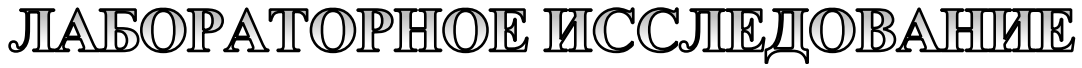

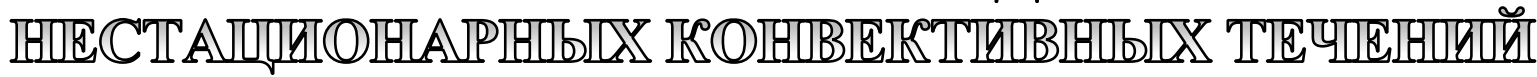

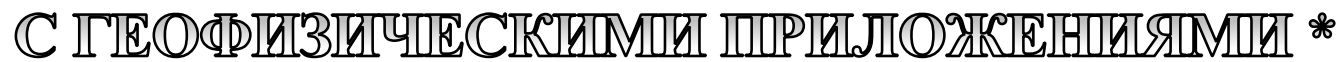

\author{
А.Н. Сухановский, Институт механики сплошных сред УрО РАН \\ А.Ю. Васильев, Институт механики сплошных сред УрО РАН \\ А.В. Евграфова, Институт механики сплошных сред УрО РАН \\ А.М. Павлинов, Институт механики сплошных сред УрО РАН \\ Е.Н. Попова, Институт механики сплошных сред УрО РАН \\ В.А. Щапов, Институт механики сплошных сред УрО РАН
}

Целью работы является экспериментальное исследование конвективных течений от локализованного источника тепла с геофизическими приложениями, в качестве которых рассмотрены лабораторный аналог тропического циклона и конвективная циркуляция на масштабе мегаполиса. Основное внимание уделено изучению роли вторичных, мелкомасштабных структур в пограничном слое на интенсификацию теплообмена. Экспериментально исследованы особенности процесса теплообмена от локализованного источника тепла в цилиндрическом слое для различных значений числа Прандтля и аспектных отношений. Проведено лабораторное исследование влияния конвективных валов на усиление теплового потока с поверхности моря и инициирования процесса быстрой интенсификации тропических циклонов. Реализован оригинальный подход обеспечивающий сопряжение измерительных систем и суперкомпьютера. Предложена и апробирована методика для лабораторного моделирования воздушных потоков и процессов теплообмена на масштабах мегаполисов.

Ключевые слова: конвекция, интенсивные вихри, лабораторное моделирование, PIV, геофизические течения.

\section{Введение}

Естественная конвекция в замкнутых полостях является классическим объектом фундаментальных исследований, притягивающим внимание разнообразием динамических режимов [1,2]. Наиболее полно изучена конвекция Релея-Бенара в полостях простой геометрии (цилиндр, параллелепипед), когда нижняя граница подогревается, а верхняя - охлаждается [3-5].
Однако многие прикладные задачи требуют рассмотрения более сложных систем, включающих конвекцию от локализованных источников тепла [6-8]. Одной из ключевых задач, связанных с конвективными течениями, является задача конвективного переноса тепла, в которой для его характеристики традиционно используется число Нуссельта (отношение полного потока тепла к теплопровод-

\footnotetext{
* Работа выполнена в рамках гранта РФФИ № 17-45-590846 - урал.
} 
ному потоку). При этом большие усилия направляются на то, чтобы выявить зависимость числа Нуссельта от основных определяющих параметров, таких как числа Релея и Прандтля. Прежде всего, это обусловлено необходимостью параметризации теплообменных процессов при очень больших значениях числа Релея, характерных для крупных промышленных установок и атмосферных процессов.

В силу того что именно конвекция является основным источником движения в геофизических системах, исследованиям конвективных течений во вращающихся слоях уделяется большое внимание. Одной из наиболее актуальных проблем динамической метеорологии является тропический циклогенез, а именно формирование интенсивных атмосферных вихрей, таких как тропические циклоны (ураганы, тайфуны), которые наносят колоссальный ущерб и приводят к человеческим жертвам. Проблема формирования тропических циклонов в целом чрезвычайно сложна, так как она сочетает в себе целый ряд гидродинамических и термодинамических процессов. Одним из важных аспектов данной проблемы является быстрая интенсификация тропических циклонов. Как показывает вышедший недавно обзор публикаций по проблемам, связанным с тропическими циклонами [9], и отчет по результатам основной конференции по тропическому циклогенезу [10], несмотря на громадные усилия и большое количество опубликованных работ эта проблема далека от своего разрешения.

Важным аспектом лабораторного моделирования геофизических течений, в частности моделирования тропических циклонов, является реализация обратной связи между теми или иными процессами в системе. Реализация управляемой обратной связи требует решения ряда технических проблем, таких как сбор и хранение данных, обработка данных в реальном времени, интеграция PIV (Particle Image Velocimetry) и систем управления нагревом. Обычный способ применения метода PIV для реконструкции скорост- ного поля состоит в получении изображений трассеров и их постобработке. Основная проблема в реализации измерений PIV в реальном времени (RTPIV) - это необходимость больших вычислительных ресурсов для постобработки данных [11].

Существует несколько способов реализации RTPIV. Упрощенные алгоритмы PIV и небольшие изображения позволяют обрабатывать изображения PIV до 15 Гц [12]. Другой способ - использование технологии FPGA (программируемая логическая матрица), к серьезным ограничениями которой требование использования конкретного аппаратного языка для программирования кода PIV. Подробное описание подхода, основанного на FPGA, можно найти в $[11,13]$. Рост вычислительной мощности GPU (графических процессоров) приводит к RTPIV на основе реализации кода GPU для обработки PIV [14]. Альтернативное эффективное решение описанной проблемы является одной из целей настоящего исследования. Ключевая идея предлагаемого подхода передача ресурсоемкой обработки данных на суперкомпьютер.

Быстрый рост мегаполисов привел к тому, что в настоящее время активно развивается область науки, направленная на исследование климатических условий на масштабе крупных городов [15], в англоязычной литературе известная как «urban climatology». Нарастающий интерес к данной проблеме подтверждается большим количеством публикаций (см. ссылки в $[16,17])$. В основном эти работы ведутся методами математического моделирования, что является чрезвычайно трудной задачей, так как самые мощные суперкомпьютеры не позволяют проводить прямое численное моделирование конвекции на масштабе городов. Поэтому приходится использовать очень серьезные упрощения, различные модельные подходы, что, безусловно, требует валидации. Поэтому новая методика, а именно применение лабораторного моделирования для изучения распространения конвективных течений в условиях реальной топографии крупных 
городов является актуальной задачей и может стать началом комплексного использования данного подхода для прогнозирования последствий различных техногенных катастроф и влияния выбросов крупных индустриальных предприятий.

Целью работы является экспериментальное исследование конвективных течений от локализованного источника тепла с геофизическими приложениями. Конкретно рассмотрено влияние вторичных течений над локализованным источником тепла на интенсивность теплообмена, проведено лабораторное моделирование нестационарной фазы лабораторного аналога тропического циклона и конвективной циркуляции на масштабе мегаполиса. Представлен оригинальный подход, позволяющий реализовать ресурсоемкие вычисления в режиме реального времени.

Данный раздел посвящен экспериментальному исследованию тепловой конвекции от локализованного источника тепла в неподвижном цилиндрическом слое. Схема установки, позволяющей проводить исследования как в неподвижном, так и во вращающемся слое представлена на рис. 1. Эксперименты проводились для широкого интервала чисел Рэлея, различных чисел Прандтля и аспектного отношения. Основной упор был сделан на изучении влияния вторичных течений в пограничном слое на процесс теплообмена. Показано, что для исследованного интервала чисел Рэлея вторичные течения (рис. 2) характеризуются значительным изменением их структуры и интенсивности. Важным результатом исследования является то, что, вопреки ожиданиям, степенной закон, описывающий зависимость числа Нуссельта от числа Рэлея, для рассматриваемой экспериментальной конфигурации не изменяется в ходе трансформации конвективных структур в пограничном слое.

Предложена физическая интерпретация результатов. Варьирование аспектного отношения также показало, что в данной конфигурации изменение аспектного отношения не влияет на степенную зависимость числа Нуссельта от числа Рэлея (рис. 3).

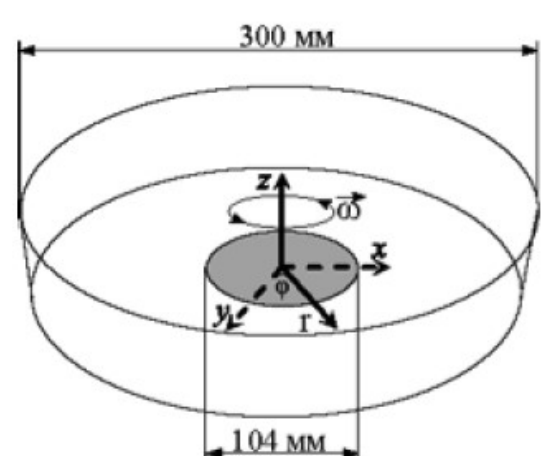

$a$

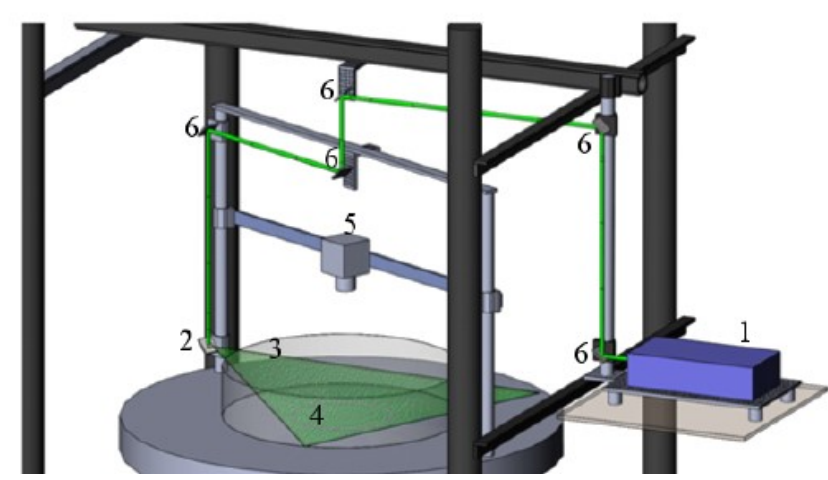

$\sigma$

Рис.1. Экспериментальная установка: а-расположение системы координат; б-схема измерения скоростей: 1 -лазер, 2 - оптическая насадка, 3 -лазерньй нож, 4 - светоотражающие частищь, 5 - цирровая камера, 6-оптические зеркала
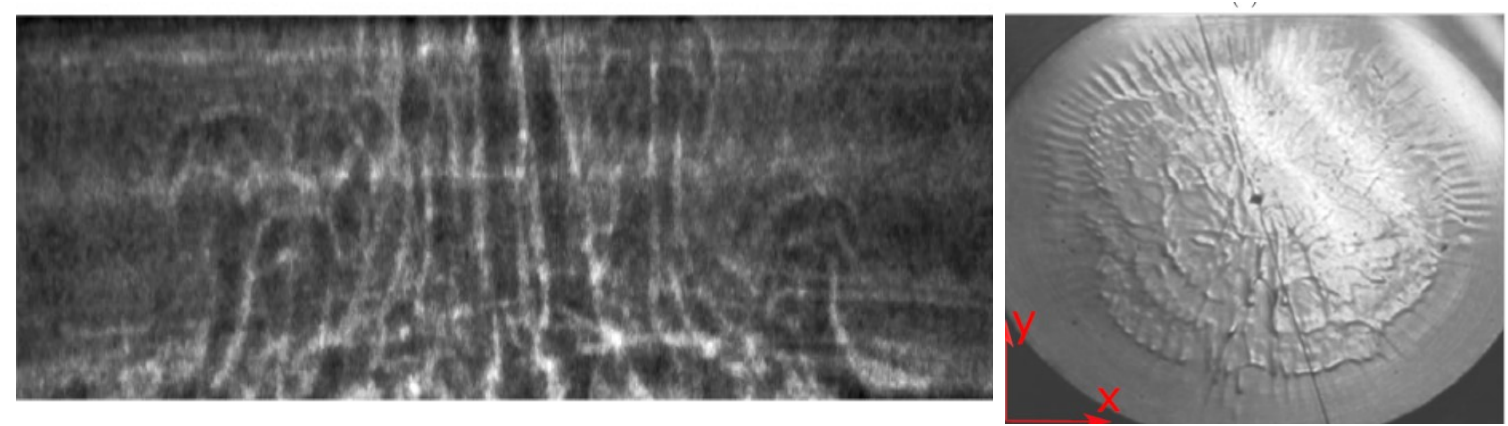

Рис. 2. Вторичные конвективные структуры над областью нагрева 


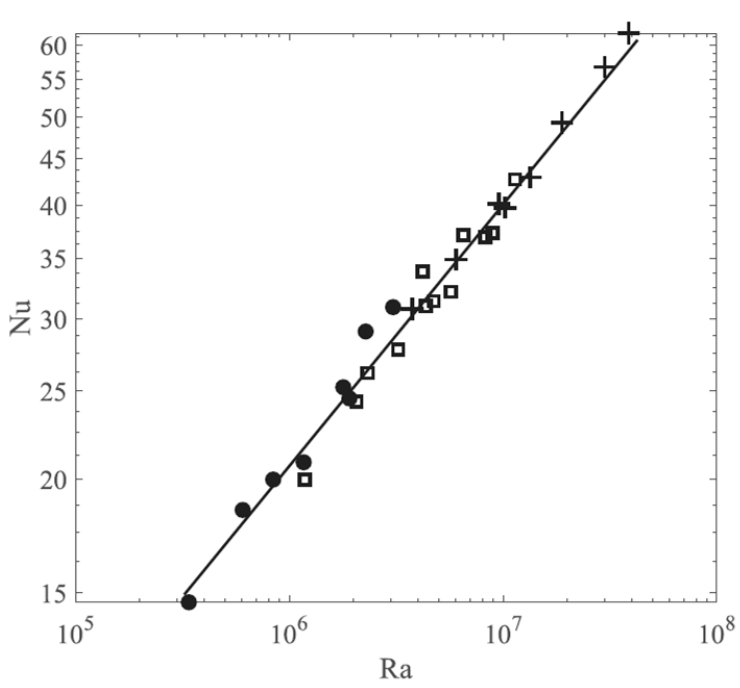

Рис. 3. Зависимость числа Нуссельта от числа Рэлея для различных жидкостей

Обнаружено, что частота формирования вторичных структур (конвективных валов) существенно зависит от аспектного отношения, на основе этого предложен безразмерный параметр, описывающий частоту формирования валов и позволивший обобщить результаты, полученные для набора аспектных отношений. Подробно полученные результаты изложены в [18].

\section{Лабораторное исследование влияния конвективных валов на усиление теплового потока}

В рамках проекта было проведено лабораторное исследование, посвященное роли конвективных валов в усилении теплового потока с поверхности моря и инициирования процесса быстрой интенсификации тропических циклонов.

Полученные результаты ясно показывают, что быстрая интенсификация лабораторного аналога тропического циклона тесно связана с процессом теплообмена в пограничном слое. Появление когерентных конвективных структур, таких как термики и валики, регистрируется различными оптическими методами и измерениями температуры. Двумерные поля скоростей используются для исследования структуры и характеристик потока.

Тепловой поток от нагретой пластины к жидкости измеряется напрямую. Образование вторичных конвективных струк- тур сильно увеличивает теплопередачу и интенсивность конвективных течений. Интенсивность радиального потока является ключевым аспектом для усиления циклонического вихря, следовательно, быстрое изменение теплообмена является фактором, который оказывает существенное влияние на динамику лабораторного вихря. Временные ряды максимальных радиальных и азимутальных скоростей находятся в хорошем качественном согласии с таковыми из теоретической модели (рис. 4). Показано, что средний квадрат скорости линейно зависит от разности температур адвективного потока и нагретой поверхности. Подробно полученные результаты изложены в [19].

\section{Применение суперкомпьютерной обработки данных для проведения экспериментов с обратной связью}

В ходе проекта реализован оригинальный подход, обеспечивающий сопряжение измерительных систем и суперкомпьютеpa $[20,21]$. Для этого была разработана технология распределенной обработки интенсивных потоков данных от экспериментальных установок на суперкомпьютерах в реальном времени. Реализованы архитектура системы распределения потока данных, программного обеспечения обработки данных и прикладной алгоритм для обработки полевых измерений скорости методом PIV. Применение технологии об-

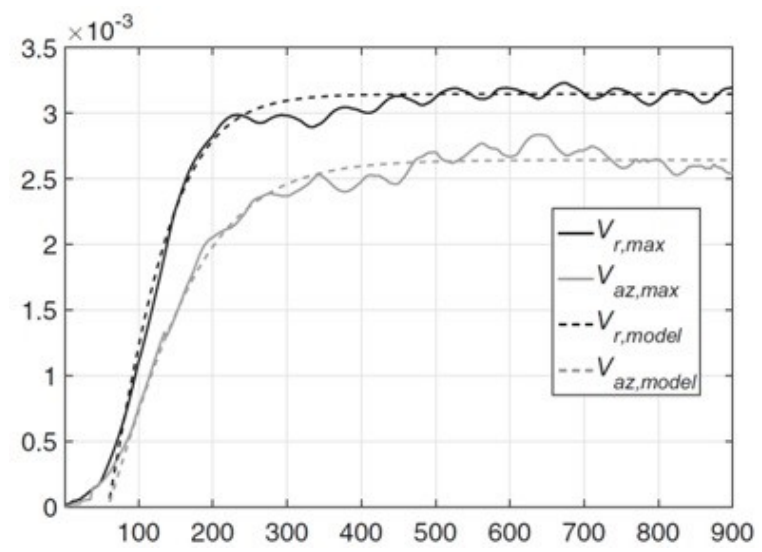

Рис. 4. Зависимость различных компонент скорости от времени, полученные в эксперименте и при помощи теоретической модели 
работки данных в реальном времени дает возможность проводить эксперименты с обратной связью, когда управляющее воздействие на систему должно зависеть от ее измеряемых внутренних параметров. Предложен подход, обеспечивающий передачу данных от измерительной системы к суперкомпьютеру через сетевое хранилище (рис. 5). Достоинствами данного подхода являются простота внедрения и высокая скорость передачи данных.

Связь измерительной системы и суперкомпьютера позволяет реализовать измерения в режиме реального времени, в том числе с обратной связью и использованием требовательных к вычислительным ресурсам алгоритмов обработки [22]. Практическое применение демонстрируется на примере экспериментального исследования формирования циклонического вихря над локализованным источником тепла при наличии связи между интенсивностью нагрева и скоростью течения [23]. Характеристики течения, полученные в результате обработки на суперкомпьютере изображений методом PIV, служат входными параметрами для системы нагрева лабораторной установки в ходе проведения измерений. Кроме того, описана возможность использования данной технологии в экспериментальном стенде для авиационной промышленности. В перспективе разработанный подход может иметь широкое применение для контроля и управления сложными системами с обратной связью.

\section{Лабораторное \\ моделирование тепловых \\ процессов на масштабе города}

Для реализации лабораторного исследования процессов теплообмена на масштабе г. Перми при помощи 3D принтера был создан макет центральной части города в масштабе 1:4 000, захватывающий набережную реки Камы. Температура измерялась при помощи массива термопар и инфракрасной камеры Flir. Часть термопар была расположена внутри и на поверхности теплообменников, на дне модели. Использование инфракрасной камеры позволило получать мгновенные полевые распределения температуры (рис. 6).

В ходе реализации проекта проведена серия измерений полей температуры в различных условиях, а именно при наличии и отсутствии внешней циркуляции. Получены данные временных вариаций пространственного распределения температуры (при помощи тепловизора) после выключения инфракрасного нагрева (рис. 7). Построены временные зависимости средней температуры от времени (рис. 8). Данное исследование направлено на моделирование остывания приземного атмосферного слоя на масштабе города, выявления его основных особенностей, прежде всего, влияния рельефа поверхности, городской застройки. Работы по данному направлению, несмотря на перспективность и актуальность, далеки от своего завершения и будут продолжены.

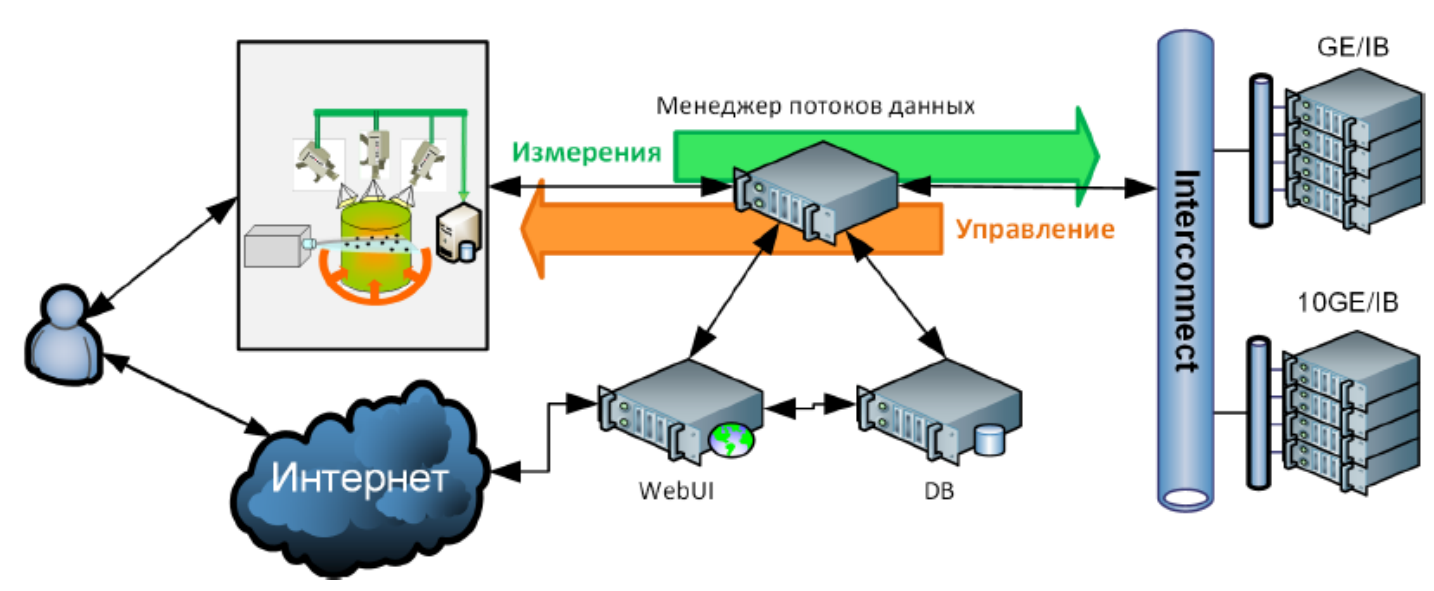

Рис. 5. Схема обработки экспериментальных данных на суперкомпьютере 


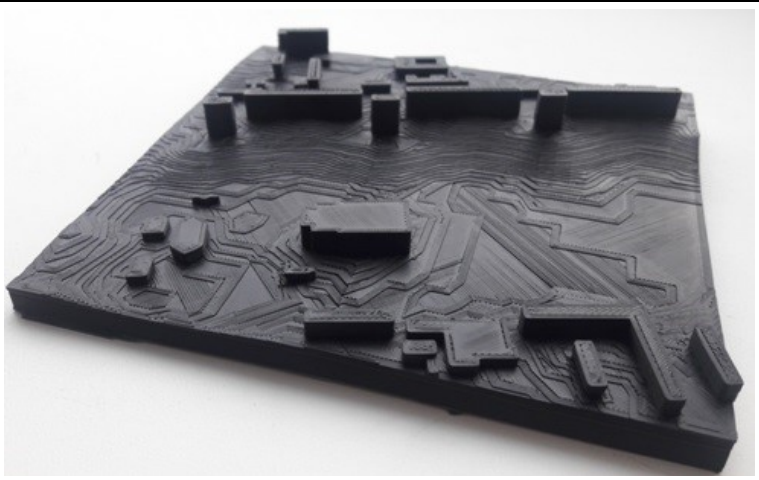

Рис. 6. Фрагмент трехмерного макета центральной части города Перми

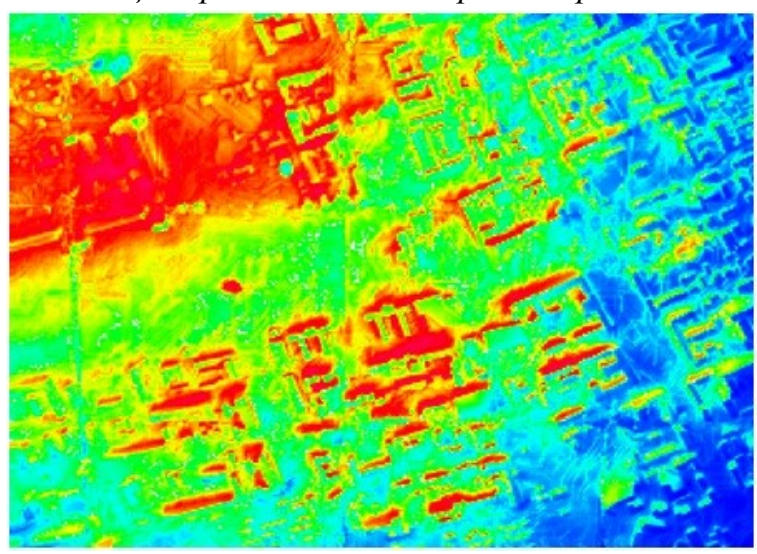

Рис. 7. Пример поля температур, полученного при помощи тепловизора

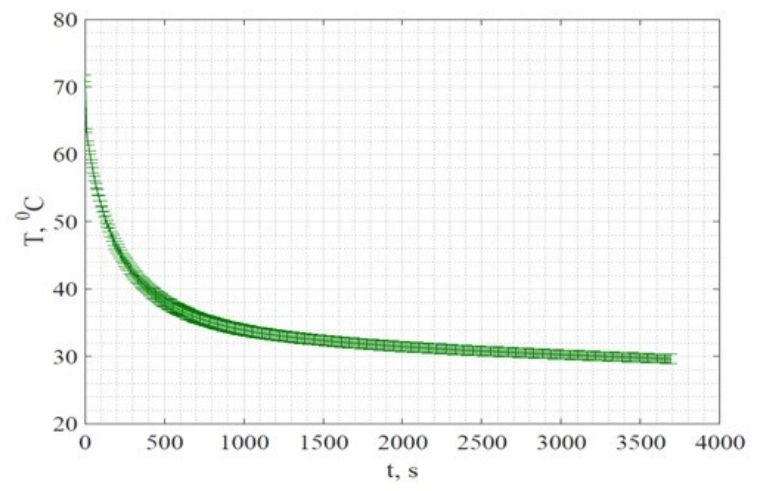

Рис. 8. Зависимость средней температурь от времени (3 реализации)

\section{Выводы}

Основной целью представленной работы было исследование конвективных течений, их эволюции и механизмов формирования, в том числе и с геофизическими приложениями. Конвективные течения существенным образом влияют на процессы теплопереноса, поэтому значительная часть работ проекта была посвящена изучению роли вторичных, мелкомасштабных структур в пограничном слое на интенсификацию теплообмена. Экспериментально исследованы особенности процесса теплообмена от локализованного источника тепла в цилиндрическом слое для различных значений числа Прандтля и аспектных отношений. Особое внимание было уделено степенным законам, описывающим зависимость числа Нуссельта от числа Рэлея. Предложена физическая интерпретация результатов.

Одной из актуальных проблем современной метеорологии, а конкретнее тропического циклогенеза, является проблема быстрой интенсификации тропических циклонов. В рамках проекта было проведено лабораторное исследование, посвященное роли конвективных валов в усилении теплового потока с поверхности моря и инициирования процесса быстрой интенсификации тропических циклонов. Полученные результаты убедительно показали, что быстрая интенсификация лабораторного аналога тропического циклона тесно связана с процессом теплообмена в пограничном слое.

Реализован оригинальный подход, обеспечивающий сопряжение измерительных систем и суперкомпьютера. Для его реализации разработана технология распределенной обработки интенсивных потоков данных от экспериментальных установок на суперкомпьютерах в реальном времени. Описаны архитектура системы распределения потока данных, программного обеспечения обработки данных и прикладной алгоритм для обработки полевых измерений скорости методом PIV. Применение технологии обработки данных в реальном времени дает возможность проводить эксперименты с обратной связью, когда управляющее воздействие на систему должно зависеть от ее измеряемых внутренних параметров, что чрезвычайно актуально для задач динамической метеорологии. В перспективе разработанный подход может иметь широкое применение для контроля и управления сложными системами с обратной связью.

Предложен и апробирован оригинальный подход для лабораторного моделиро- 
вания воздушных потоков и процессов теплообмена на масштабах мегаполисов. Для его реализации при помощи 3D-принтера был создан макет центральной части города Перми в масштабе 1:4 000, захва- тывающий набережную реки Камы. В ходе реализации проекта проведена серия измерений полей температуры в различных условиях, а именно при наличии и отсутствии внешней циркуляции.

\section{Библиографический список}

1. Гершуни Г.З., Жуховицкий Е.М., Непомнящий А.А. Устойчивость конвективных течений // - М.: Наука, 1989. 320 с.

2. Зимин В.Д., Фрик П.Г. Турбулентная конвекция // М.: Наука, 1988. - 178 с.

3. Siggia E.D. High Rayleigh number convection // Annu. Rev. Fluid Mech., 1994. - Vol. 26. - P. $137-168$.

4. Ahlers G., Grossmann S., Lohse D. Heat transfer and large-scale dynamics in turbulent Rayleigh-Benard convection // Rev. Mod. Phys. - 2009. - Vol. 81. - P. 503-537.

5. Chilla F., Schumacher J. New perspectives in turbulent Rayleigh-Benard convection // Eur. Phys. J.E. 2012. - Vol. 35, 58.

6. Oztop H.F., Estelle P., Yan W.-M., Al-Salem K., Orfi J., Mahian O. A brief review of natural convection in enclosures under localized heating with and without nanofluids // Int. Comm. Heat Mass Tran., - 2015. Vol. 60. - P. 37-44.

7. Sukhanovskii A., Evgrafova A., Popova E. Horizontal rolls over localized heat source in a cylindrical layer// Phys. Nonlinear Phenom. - 2016. - Vol. 316. - P. 23-33.

8. Bakhuis D., Ostilla-Monico R., van der Poel E.P., Verzicco R., Lohse D. Mixed insulating and conducting thermal boundary conditions in Rayleigh-Benard convection // J. Fluid Mech. - 2018. - Vol. 835. - P. 491-511.

9. Emanuel K. 100 years of progress in tropical cyclone research // Meteorol. Monogr. 59:15-1. (2018), https://doi.org/10.1175/AMSMONOGRAPHS-D-18-0016.1.

10. Vigh J.L. Tropical cyclone intensity change: internal influences-rapporteur report, topic $3.1 / /$ Conference paper IWTC-9. - 2018. - P. 1-72.

11. Kreizer, M., Ratner, D., Liberzon, A. Real-time image processing for particle tracking Velocimetry // Exp. Fluids 48(1). - P. 105-110 (2010).

12. Willert C.E., Munson M.J., Gharib M. Real-time particle image velocimetry for closed-loop flow control applications // In: 15th International Symposium on Applications of Laser Techniques to Fluid Mechanics (2010).

13. Yu H., Leeser M., Tadmor G., Siegel S. Real-time particle image velocimetry for feedback loops using FPGA implementation // J. Aerosp. Comput. Inf., Commun. - 2006. - Vol. 3(2). - P. 52-62.

14. Gautier N., Aider J.L. Real-time planar flow velocity measurements using an optical flow algorithm implemented on GPU // J. Vis. - 2015. - Vol. 18(2). - P. 277-286.

15. Arnfield, A.J. Two decades of urban climate research: a review of turbulence, exchanges of energy and water, and the urban heat island // Int. J. Climatol., - 2003. - Vol. 23: 1-26. doi:10.1002/joc.859.

16. Sessa V., Xie Z.T., Herring S. Thermal stratification effects on turbulence and dispersion in internal and external boundary layers // Boundary-Layer Meteorology. - 2019.

17. Grylls T. [et al.] Steady-State Large-Eddy Simulations of Convective and Stable Urban Boundary Layers // Boundary Layer Meteorology. - 2020. - T. 175. - №. 3. - C. 309-341.

18. Evgrafova A., Sukhanovskii A. Specifics of heat flux from localized heater in a cylindrical layer // International Journal of Heat and Mass Transfer. - 2019. - T. 135. - C. 761-768.

19. Sukhanovskii A., Popova E. The Importance of Horizontal Rolls in the Rapid Intensification of Tropical Cyclones // Boundary-Layer Meteorology. - 2020. - C. 1-18. https://doi.org/10.1007/s10546-020-00503-2.

20. Щапов В.А., Евграфова А.В., Масич Г.Ф. [и др.] Применение суперкомпьютерной обработки данных от измерительных систем для проведения экспериментов с обратной связью // Программные системы: теория и приложения, - 2018, - Vol. 9:1(36), С. 3-19.

21. Stepanov R., Sozykin A. Distributed PIV Technology: Network Storage Usage // CEUR Workshop Proceedings. - CEUR-WS, - 2017. - T. 1990. - C. 121-129.

22. Sukhanovskii A.,Shchapov V., Pavlinov A., Popova E. Laboratory model of tropical cyclone with controlled forcing // Journal of Physics: Conference Series, - 2018/ - Vol. 1128. - 012133, doi: https://doi.org/10.1088/1742-6596/1128/1/012133.

23. Sukhanovskii A. [et al.] Different aspects of laboratory analog of tropical cyclone // IOP Conference Series: Earth and Environmental Science. - IOP Publishing, 2019. - T. 231. - №. 1. - C. 012052. 


\title{
LABORATORY STUDY OF NON-STATIONARY CONVECTIVE FLOWS
} WITH GEOPHYSICAL APPLICATIONS

\author{
A.N. Sukhanovskii, A.Yu. Vasiliev, A.V. Evgrafova, A.M. Pavlinov, \\ E.N. Popova, V.A. Shchapov
}

Institute of Continuous Media Mechanics UB RAS

A study of convective flows, their evolution and formation mechanism has been carried out. Convective flows significantly affect the processes of heat transfer; therefore, the main attention is paid to the studying the role of secondary, small-scale structures in the boundary layer for the intensification of heat transfer. The features of the heat transfer process from a localized heat source in a cylindrical layer for various values of the Prandtl number, as well as the aspect ratios, are experimentally investigated. A laboratory study of the effect of convective rolls on the enhancement of the heat flux from the sea surface and the initiation of of rapid intensification process of tropical cyclones was carried out. An original approach has been implemented that combines measuring systems and a supercomputer. An approach for laboratory modeling of air flows and heat transfer processes on the scale of a metropolis has been proposed and tested.

Keywords: convection, intensive vortices, laboratory modeling, PIV, geophysical flows.

\section{Сведения об авторах}

Сухановский Андрей Николаевич, кандидат физико-математических наук, старший научный сотрудник, Институт механики сплошных сред УрО РАН - филиал Пермского федерального исследовательского центра УрО РАН (ИМСС УрО РАН), 614013, ул. Академика Королева, 1; e-mail:san@icmm.ru

Васильев Андрей Юрьевич, кандидат физико-математических наук, научный сотрудник, ИМСС УрО PAH; e-mail: vasand@math.nsc.ru

Евграфова Анна Валерьевна, кандидат физико-математических наук, младший научный сотрудник, ИМСС УрО РАН; e-mail: eav@icmm.ru

Павлинов Александр Михайлович, кандидат физико-математических наук, младший научный сотрудник, ИМСС УрО РАН; e-mail: pam@icmm.ru

Попова Елена Николаевна, кандидат физико-математических наук, научный сотрудник, ИМСС УpО РАН; e-mail: popovadu@icmm.ru

Щапов Владислав Алексеевич, кандидат технических наук, младший научный сотрудник, ИМСС УpO РАН; e-mail: shchapov@icmm.ru 